\title{
Intrapleural permanent pacemakers in infancy
}

\author{
M HICKEY, D DUFF, AND M C NELIGAN \\ Department of Cardiac Surgery, Our Lady's Hospital for Sick Children, Crumlin, Dublin
}

SUMMARY The pleural cavity offers a suitable site for permanent pacemaker implantation in infancy. We describe 3 infants in whom this approach was successfully used.

Although treatment with permanent pacemakers has been extensively described in adults, there is little information about it in infants. Conventional sites for pacemaker implantation are the abdominal wall, subpectoral muscle region, subdiaphragmatic area, and the pelvic cavity. If the infant has a thin chest and abdominal wall, implantation of the pacemaker there produces an unsightly bulge and there is the ever present threat of wound dehiscence or wound infection because of the fairly large size of the unit. ${ }^{1}$ We describe our experience with permanent pacemaker implants in 3 infants using an intrapleural site.

\section{Case reports}

Case 1. A girl was born with clinical signs of congestive cardiac failure. Her heart rate was 35 to 40 beats a minute and she had a murmur characteristic of a patent ductus arteriosus. Her electrocardiograph showed complete heart block and numerous premature ventricular contractions. Cardiac catheterisation was performed within 24 hours of birth. A patent ductus arteriosus was confirmed but there was no other anatomical abnormality. A temporary transvenous pacemaker was placed in her right ventricle. When she was 6 days old a multiprogrammable permanent pacemaker was implanted into the left pleural cavity through an anterior thoracotomy. Her weight at operation was $3.39 \mathrm{~kg}$. The postoperative recovery was complicated by a left upper lobe pneumonia causing considerable respiratory distress. This responded during the course of several days to vigorous physiotherapy and treatment with appropriate antibiotics. Mild respiratory distress persisted for 1-2 months. The baby was discharged from hospital after 24 days. She is now 2 years old and thriving. No difficulty has been experienced with reprogramming.

Case 2. A boy was born with a patent ductus arteriosus murmur; his electrocardiograph showed complete heart block. At age 3 months he developed congestive cardiac failure. His ventricular rate was 38 beats a minute and he was transferred to our hospital for further treatment. Cardiac catheterisation confirmed the presence of a patent ductus arteriosus and a temporary pacemaker was inserted at this procedure. A permanent programmable pacemaker was then implanted through a left thoracotomy. The age at operation was 3 months and the weight was $4.34 \mathrm{~kg}$. His patent ductus was ligated at surgery. His postoperative recovery was protracted and he was slow to thrive showing a pronounced reluctance to feed. However, there was no clinical or radiological evidence of pneumonia at any stage. He was discharged from hospital one month later. He was readmitted at age 9 months because his pacemaker was failing to capture. The pacing threshold had risen above the capability of the unit and he required re-exploration of the pacemaker and lead. The lead was resited and he is now well.

Case 3. A 1-year-old girl presented with evidence of congestive cardiac failure complicated by StokesAdams attacks. The electrocardiograph showed complete heart block with a ventricular rate of 38 beats a minute. A temporary transvenous pacemaker was inserted. A more detailed cardiac catheterisation was not performed because of her critical state at that time. A multiprogrammable pacemaker* was then implanted through a left anterior thoracotomy and the electrode attached to the left ventricular myocardium. The baby's weight at operation was $5.0 \mathrm{~kg}$. She was discharged from hospital 10 days after surgery. Now, more than 2 years later, she remains well and is maintained on digoxin only. From time to time her pacemaker has been reprogrammed. This was performed without difficulty and there has been no decrease in sensitivity.

*The pacemaker used in each case was an Arco Unipolar Type B with an epicardial lead. 


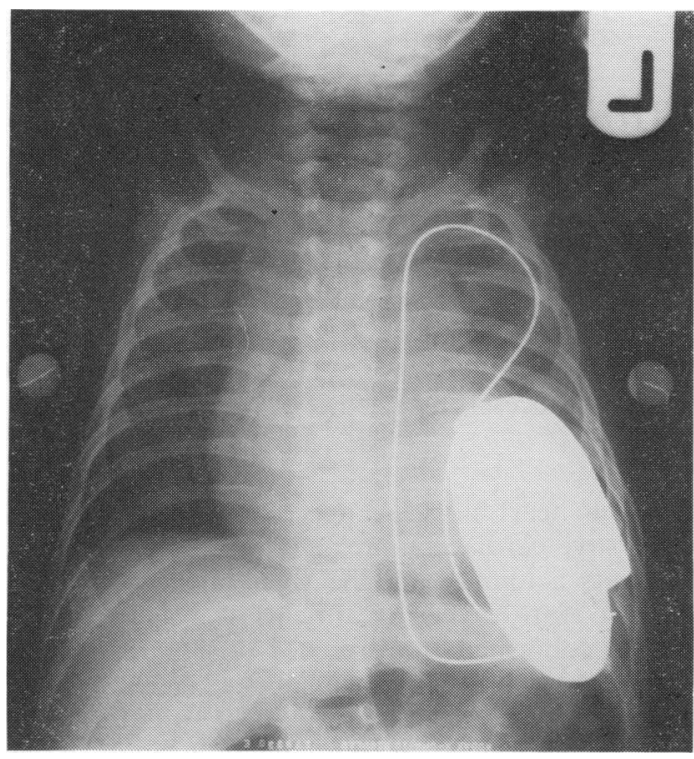

Figure Case 2, three weeks after initial implant.

\section{Discussion}

The purpose of this report is to illustrate the use of cardiac pacemakers implanted within the pleural cavity in small babies. Intrapleural pacemaker implantation in children has previously been reported but hitherto this technique has not been described in infancy. ${ }^{2-4}$

There are certain criteria that should be adhered to. The pacemaker must be positioned in a site that will allow healing; it must be accessible for easy reprogramming and for future generator or lead replacement; it should be secure from trauma and be cosmetically acceptable. Problems related to growth cannot be avoided when dealing with young patients.

Positioning the pacemaker within the pleural cavity has advantages. Even in small babies the chest cavity is large enough to accommodate the pacemaker without difficulty (Figure). A limited thoracotomy is an easy procedure at this age and is well tolerated. The left side is the more appropriate as it allows easier attachment of the pacing lead to the myocardium. The pacemaker is placed within the pleural cavity and the expanding lung anchors it to the parietal pleura. Fibrosis subsequently secures it in position. Migration of the pacemaker has not been a problem in our experience. Reprogramming when required has been performed without difficulty.
Re-exploration was necessary in one of our? patients. In this case the baby presented 6 months? after his original surgery. The problem was failure of ventricular capture despite normal generator function. Radiographical investigation showed no듬 sign of lead fracture or detachment. Because of the $\frac{\overline{\bar{n}}}{\mathrm{n}}$ high pacing threshold required to achieve even $\vec{\sigma}_{\bar{\sigma}}$ intermittent capture the electrode was resited. At $\mathrm{O}$ operation the pacemaker was easily exposed by aw direct intercostal incision. It was firmly encased $\vec{\circ}$ within a fibrous sac and was readily accessible:should replacement of the generator have been $\vec{\omega}$ required. The lead was resited in the myocardiumo and pacing resumed satisfactorily. The baby'soํㅇ postoperative recovery was uneventful. This oper-cr ation illustrated the ease of access in small patients. for replacement of intrapleural pacemakers.

Intrapleural pacemakers are cosmetically more $\stackrel{N}{\mathcal{N}}$ acceptable than those implanted subcutaneously.을 Unsightly bulges and the risk of wound dehiscence are avoided and the unit is protected from trauma.

Generator failure and lead failure continue to be problems. Regardless of where the pacemaker is $\vec{\bullet}$ sited regular surveillance and reprogramming in the ${ }_{N}^{\infty}$ pacemaker clinic are mandatory.

The siting of pulse generators within the pleuralo cavity for artificial cardiac pacing in infancy is well tolerated even by the newborn and has many advantages compared with more standard $\frac{0}{\mathbb{Q}}$ approaches.

\section{References}

1 Liu L, Griffiths S P Gerst P $\mathrm{H}$. Implanted cardiac pacemakers in children. A report of their application in five patients. Am J Cardiol 1967; 20: 639-47.

${ }^{2}$ Escano F B, Jr, Berroya R B, Gianfrancesco H, Macasaet R A, Khicha G C J. Intrapleural pacemaker 3 . generator in children.J Thorac Cardiovasc Surg 1971; 62: 454-6.

${ }^{3}$ Lindesmith G G, Stiles Q R, Meyer B W, Jones J C, Stanton R E. Experience with an implantable synchronous 윽 pacemaker in children. Ann Thorac Surg 1968; 6: 358-64.

4 Kahn D R, Stern A, Sigman J, Sloan H. An emergency method of handling broken pacemaker wires in children. Am J Cardiol 1965; 15: 404-6.

Correspondence to $\operatorname{Dr} M$ Hickey, Our Lady's Hospital for Sick Children, Crumlin, Dublin 12, Ireland.

Received 7 April 1982 